\title{
Clinical utility gene card for: DPAGT1 defective congenital disorder of glycosylation
}

\author{
Jaak Jaeken ${ }^{\star, 1}$, Dirk Lefeber ${ }^{2}$ and Gert Matthijs ${ }^{3}$ \\ European Journal of Human Genetics (2015) 23, doi:10.1038/ejhg.2015.177; published online 5 August 2015
}

\section{DISEASE CHARACTERISTICS}

1.1 Name of the disease (synonyms)

Deficiency of UDP-GlcNAc:Dol-P-GlcNAc-P transferase 1, deficiency of Dol-P:GlcNAc-P transferase 1, deficiency of GlcNAc-1-P transferase 1, congenital myasthenic syndrome with tubular aggregates 2, CMSTA2, DPAGT1-CDG, CDG-Ij.

\subsection{OMIM\# of the disease}

608093.

614750

1.3 Name of the analysed genes or DNA/chromosome segments DPAGT1

\subsection{OMIM\# of the gene(s)}

191350.

\subsection{Mutational spectrum}

Twenty-five variants have been reported: twenty-one missense variants, three splicing variants and one duplication vari ant $^{1-11}$ (www.lovd.nl/DPAGT1). The standard reference sequence indicating reported variants (ENSG00000172269) and a reference for exon numbering (ENST00000354202) can be found at http://www. ensembl.org.

\subsection{Analytical methods}

Sanger sequencing of the nine coding exons and flanking intronic sequences of the DPAGT1 gene (NCBI reference sequence: NM_000011.10).

\subsection{Analytical validation}

Sanger sequencing identifies variants in $>99 \%$ of patients. Deep intronic variants, large deletions and duplications would not be detected using this approach. Novel variants with uncertain pathogenic nature are of course possible.

\subsection{Estimated frequency of the disease}

(Incidence at birth ('birth prevalence') or population prevalence)

If known to be variable between ethnic groups, please report:
Forty-one patients (from seventeen families) have been reported. ${ }^{1-15}$ The frequency and the prevalence of the disease are not known.

\subsection{Diagnostic setting}

\begin{tabular}{lll}
\hline & Yes & No \\
A. (Differential) diagnostics & $\bigotimes$ & $\square$ \\
B. Predictive testing & $\bigotimes$ & $\square$ \\
C. Risk assessment in relatives & $\bigotimes$ & $\square$ \\
D. Prenatal & $\bigotimes$ & $\square$
\end{tabular}

Comment: DPAGT1-CDG is an autosomal recessive disease that presents as one of the two different phenotypes: an encephalopathy in the context of a multisystem disorder ${ }^{1,2,4-7,9,13}$ and, on the other hand, a congenital myasthenic syndrome. ${ }^{3,8,10-12,14,15}$ The multisystem presentation (28 patients) is, as a rule, a severe disease. Twenty-three patients died between 6 weeks and 5 years. Two siblings had a milder presentation, and were 34 and 32 years old when reported. All patients showed moderate to severe psychomotor disability, and most patients had microcephaly, hypotonia, and epilepsy. Less frequent symptoms were feeding difficulties, apnoea, respiratory insufficiency, chronic anaemia, cataracts, hypotrophy, hypertrichosis, frequent episodes of aspiration, hypertonia of the extremities, hypo- and hyperreflexia, joint contractures, and abnormal brain magnetic resonance imaging (MRI). Features reported only once included a foetal hypokinesia syndrome, tremor, night blindness, inverted nipples, fat pads, skin dimples on thighs, bilateral papillar atrophy, bilateral cochlear impairment, fiber type disproportion on muscle biopsy, and abnormal brain PET scan. Biochemical abnormalities included increased serum transaminases (4 patients) and creatine kinase (1 patient), hypoproteinemia (1 patient), decreased antithrombin (1 patient), and a type 1 pattern on serum transferrin isoelectrofocusing in all patients. The congenital myasthenic syndrome presentation has been reported in 13 patients. The first symptoms were noted between birth and 17 years. Ages at report ranged from 6 to 58 years. One patient died at 26 years from a respiratory crisis. Clinical features included a predominantly proximal muscle weakness with absent or minimal craniobulbar symptoms. The syndrome was mostly slowly progressive but some patients showed mild improvement in childhood or adolescence. All

\footnotetext{
${ }^{1}$ Department of Development and Regeneration, Centre for Metabolic Disease, University Hospital Gasthuisberg, KU Leuven, Leuven, Belgium; ${ }^{2}$ Department of Neurology, Translational Metabolic Laboratory, Radboudumc, Nijmegen, The Netherlands; ${ }^{3}$ Department of Human Genetics, Centre for Human Genetics, KU Leuven, Leuven, Belgium ${ }^{*}$ Correspondence: Professor J Jaeken, Department of Development and Regeneration, Centre for Metabolic Disease, University Hospital Gasthuisberg, KU Leuven, Herestraat 49, Leuven BE 3000, Belgium. Tel: +32 16343 827; Fax: +32 16343 842; E-mail: jaak.jaeken@med.kuleuven.be

Received 23 April 2015; accepted 30 June 2015; published online 5 August 2015
} 
patients responded favourably to acetylcholinesterase inhibitors such as pyridostigmine. Muscle cramps, difficulty in swallowing and chewing, and scoliosis have been reported in a few patients, as well as delayed motor development and intellectual disability. Serum creatine kinase levels were normal. Hypoglycosylation of serum transferrin was present in only $4 / 8$ patients. Electromyography showed myopathic features but not always of the facial muscles. A frequent sign was a decremental response on 3-Hertz repetitive nerve stimulation. Tubular aggregates in muscle biopsy were noted in 4/7 patients (not in early biopsies). Analyses of motor end plates demonstrated a reduction of end plate acetylcholine receptors. Brain MRI, performed in a few patients, was normal. It has to be noted that three other congenital disorders of glycosylation can cause a congenital myasthenic syndrome: GFPT1-CDG (16), ALG2-CDG (17), and ALG14CDG (17).

The diagnosis has to be confirmed by mutation analysis of DPAGT1. An upcoming strategy is to analyse a panel of genes known to be involved in CDG and, when this is 'negative', to perform whole-exome sequencing. The identification of the variant(s) that affect(s) function will permit heterozygote detection in the family as well as prenatal diagnosis.

\section{TEST CHARACTERISTICS}

\begin{tabular}{|c|c|c|c|c|}
\hline & \multirow{2}{*}{\multicolumn{2}{|c|}{ Genotype or disease }} & \multirow{3}{*}{$\begin{array}{l}\text { A: True positives } \\
\text { B: False positives }\end{array}$} & \multirow{3}{*}{$\begin{array}{l}\text { C: False negative } \\
\text { D: True negative }\end{array}$} \\
\hline & & & & \\
\hline & Present & Absent & & \\
\hline \multicolumn{5}{|l|}{ Test } \\
\hline \multirow[t]{2}{*}{ Positive } & \multirow[t]{2}{*}{ A } & \multirow[t]{2}{*}{ B } & Sensitivity: & $A /(A+C)$ \\
\hline & & & Specificity: & $D /(D+B)$ \\
\hline \multirow[t]{2}{*}{ Negative } & \multirow[t]{2}{*}{ C } & \multirow[t]{2}{*}{$\mathrm{D}$} & Positive predictive value: & $A /(A+B)$ \\
\hline & & & Negative predictive value: & $\mathrm{D} /(\mathrm{C}+\mathrm{D})$ \\
\hline
\end{tabular}

\subsection{Analytical sensitivity}

(proportion of positive tests if the genotype is present)

Close to $100 \%$ for the multisystem presentation when using the serum transferrin isoelectrofocusing test. In the congenital myasthenic syndrome presentation, this test was positive in only about half of the reported patients.

\subsection{Analytical specificity}

(proportion of negative tests if the genotype is not present)

Close to $100 \%$ when using the serum transferrin isoelectrofocusing test. This test can be positive in secondary glycosylation disturbances such as galactosemia and hereditary fructose intolerance, and due to bacterial sialidase. ${ }^{16-18}$

\subsection{Clinical sensitivity}

(proportion of positive tests if the disease is present)

The clinical sensitivity can be dependent on variable factors such as age or family history. In such cases a general statement should be given, even if a quantification can only be made case by case.

Close to $100 \%$ for the multisystem presentation but only around $50 \%$ for the congenital myasthenic syndrome presentation.

\subsection{Clinical specificity}

(proportion of negative tests if the disease is not present)

The clinical specificity can be dependent on variable factors such as age or family history. In such cases a general statement should be given, even if a quantification can only be made case by case.

Close to $100 \%$.
2.5 Positive clinical predictive value

(life time risk to develop the disease if the test is positive)

$100 \%$, based on a positive serum transferrin isoelectrofocusing screening and DPAGT1 mutation analysis.

\subsection{Negative clinical predictive value}

(probability of not developing the disease if the test is negative)

Assume an increased risk based on family history for a nonaffected person. Allelic and locus heterogeneity may need to be considered.

Index case in that family had been tested:

$100 \%$ for the multisystem presentation, and around 50\% for the congenital myasthenic syndrome presentation.

Index case in that family had not been tested:

$100 \%$ for the multisystem presentation, and around 50\% for the congenital myasthenic syndrome presentation.

\section{CLINICAL UTILITY}

3.1 (Differential) diagnostics: The tested person is clinically affected (To be answered if in 1.9 'A' was marked)

\subsubsection{Can a diagnosis be made other than through a genetic test?}

\begin{tabular}{lll}
\hline No (continue with 3.1.4) & $\square$ & \\
Yes & $\bigotimes$ & $\square$ \\
& Clinically & $\square$ \\
& Imaging & $\square$ \\
& Endoscopy & $\square$ \\
& Biochemistry & $\square$ \\
& Electrophysiology & \\
& Other (please describe) &
\end{tabular}

\subsubsection{Describe the burden of alternative diagnostic methods to the} patient

The blood sampling for the serum transferrin isoelectrofocusing screening test and that for the mutation analysis is a minor burden to the patient.

\subsubsection{How is the cost effectiveness of alternative diagnostic methods to} be judged?

It differs among countries. In Belgium and the Netherlands the cost of these tests is largely carried by the national assurance organism.

\subsubsection{Will disease management be influenced by the result of a genetic test?}

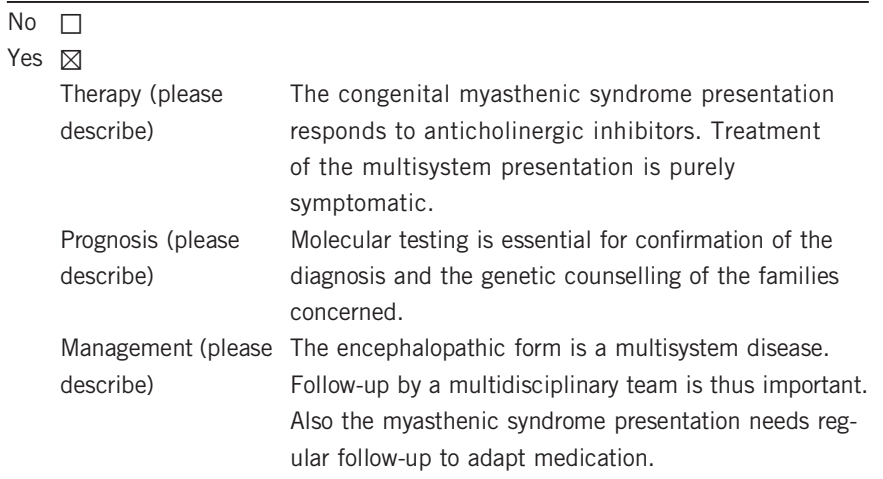


3.2 Predictive Setting: The tested person is clinically unaffected but carries an increased risk based on family history

(To be answered if in 1.9 'B' was marked)

\subsubsection{Will the result of a genetic test influence lifestyle and prevention?}

If the test result is positive (please describe): Not applicable.

If the test result is negative (please describe): Not applicable.

3.2.2 Which options in view of lifestyle and prevention does a person at-risk have if no genetic test has been done (please describe)?

Not applicable.

3.3 Genetic risk assessment in family members of a diseased person (To be answered if in $1.9^{~ ' C}$ ' was marked)

3.3.1 Does the result of a genetic test resolve the genetic situation in that family?

Usually yes, by testing the potential heterozygous persons (carriers) in the family.

3.3.2 Can a genetic test in the index patient save genetic or other tests in family members?

No.

3.3.3 Does a positive genetic test result in the index patient enable a predictive test in a family member?

Not applicable.

\subsection{Prenatal diagnosis}

(To be answered if in 1.9 'D' was marked)

\subsubsection{Does a positive genetic test result in the index patient enable a} prenatal diagnosis?

Yes. Prenatal diagnosis should be performed by molecular analysis; foetal transferrin isoelectrofocusing leads to false results. ${ }^{19}$

\section{IF APPLICABLE, FURTHER CONSEQUENCES OF TESTING}

Please assume that the result of a genetic test has no immediate medical consequences. Is there any evidence that a genetic test is nevertheless useful for the patient or his/her relatives? (Please describe).

Knowledge of the diagnosis will stop unnecessary further investigations. It will also help the parents in the process of accepting the disease, although no curative treatment is yet available.

\section{CONFLICT OF INTEREST}

The authors declare no conflict of interest.

\section{ACKNOWLEDGEMENTS}

This work was supported by EuroGentest2 (Unit 2: 'Genetic testing as part of health care'), a Coordination Action under FP7 (Grant Agreement Number 261469) and the European Society of Human Genetics.

1 Wu X, Rush JS, Karaoglu D et al: Deficiency of UDP-GlcNAc:dolichol phosphate $\mathrm{N}$-acetylglucosamine-1 phosphate transferase (DPAGT1) causes a novel congenital disorder of glycosylation Type lj. Hum Mutat 2003; 22: 144-150.

2 Vuillaumier-Barrot S: Diagnostic moléculaire des anomalies congénitales de la glycosylation. Ann Biol Clin 2005; 63: 135-143.

3 Belaya K, Finlayson S, Slater CR et al: Mutations in DPAGT1 cause a limb-girdle congenital myasthenic syndrome with tubular aggregates. Am J Hum Genet 2012; 91: 193-201.

4 Carrera IA, Matthijs G, Perez B, Cerdá CP: DPAGT1-CDG: Report of a patient with fetal hypokinesia phenotype. Am J Med Genet A 2012; 158A: 2027-2030.

5 Imtiaz F, Al-Mostafa A, Al-Hassnan A: Further delineation of the phenotype of congenital disorder of glycosylation DPAGT1-CDG (CDG-Ij) identified by homozygosity mapping. JIMD Rep 2012; 2: 107-111.

6 Timal S, Hoischen A, Lehle L et al: Gene identification in the congenital disorders of glycosylation type I by whole-exome sequencing. Hum Mol Genet 2012; 21: 4151-4161.

7 Würde AE, Reunert J, Rust S et al: Congenital disorder of glycosylation type Ij (CDG-Ij, DPAGT1-CDG): extending the clinical and molecular spectrum of a rare disease. Mol Genet Metab 2012; 105: 634-641.

8 Basiri K, Belaya K, Liu WW, Maxwell S, Sedghi M, Beeson D: Clinical features in a large Iranian family with a limb-girdle congenital myasthenic syndrome due to a mutation in DPAGT1. Neuromuscul Disord 2013; 23: 469-472.

9 Iqbal Z, Shahzad M, Vissers LELM et al: A compound heterozygous mutation in DPAGT1 results in a congenital disorder of glycosylation with a relatively mild phenotype. Eur J Hum Genet 2013; 21: 844-849.

10 Klein A, Robb S, Rushing E, Liu WW, Belaya K, Beeson D: Congenital myasthenic syndrome caused by mutations in DPAGT1. Neuromuscul Disord 2014; 25: 253-256.

11 Selcen D, Shen X-M, Brengman J et al: DPAGT1 myasthenia and myopathy. Neurology 2014; 82: 1822-1830.

12 Slater CR, Fawcett PR, Walls TJ et al: Pre- and post-synaptic abnormalities associated with impaired neuromuscular transmission in a group of patients with 'limb-girdle myasthenia'. Brain 2006; 129: 2061-2076.

13 Adamowicz M, Chmielinska E, Kaluzny $L$ et al: Clinical and biochemical characterization of the second CDGIJ (DPAGT1-CDG) patient. J Inherit Metab Dis 2011; 34: S181.

14 Belaya K, Finlayson S, Cossins J et al: Identification of DPAGT1 as a new gene in which mutations cause a congenital myasthenic syndrome. Ann NY Acad Sci 2012; 1275: 29-35.

15 Finlayson S, Palace J, Belaya K et al: Clinical features of congenital myasthenic syndrome due to mutations in DPAGT1. J Neurol Neurosurg Psychiatry 2013; 84: 1119-1125

16 Jaeken J, Pirard M, Adamowicz M, Pronicka E, Van Schaftingen E: Inhibition of phosphomannose isomerase by fructose 1-phosphate: an explanation for defective $\mathrm{N}$-glycosylation in hereditary fructose intolerance. Pediatr Res 1996; 40: 764-766.

17 Sturiale L, Barone R, Fiumara A et al: Hypoglycosylation with increased fucosylation and branching of serum transferrin N-glycans in untreated galactosemia. Glycobiology 2005; 15: 1268-1276.

18 Lefeber DJ, Morava E, Jaeken J: How to find and diagnose a CDG due to defective N-glycosylation. J Inherit Metab Dis 2011; 34: 849-852.

19 Matthijs G, Schollen E, Cassiman JJ et al: Prenatal diagnosis in CDG 1 families: beware of heterogeneity. Eur J Hum Genet 1998; 6: 99-104. 